Communications in Physics, Vol.21, No. 1 (2011), pp. 71-76

\title{
INFLUENCE OF PRINCIPLE PARAMETERS ON THE AVERAGE STIFFNESS OF OPTICAL TWEEZER USING PULSED GAUSSIAN BEAMS
}

\author{
HO QUANG QUY \\ Journal of Military Sciences and Technology \\ HOANG DINH HAI \\ Nghe An Pedagogical College \\ HOANG VAN NAM \\ Ha Tinh Peoples Committee
}

\begin{abstract}
In this article expresions used to simulate the trap stiffness $k$ of the optical trap are derived. The influence of principle parameters as total energy, beam waist and duration of pulsed laser beam, radius of dielectric particle, and viscosity of surrounding medium on the stiffness is simulated and discussed.
\end{abstract}

\section{INTRODUCTION}

A particle trapped in an optical trap is not completely immobile. There are thermal fluctuations that cause the particle to execute Brownian motion while trapped. It is the fact that the trap has an inherent stiffness $\mathrm{k}$, which is controlled by power of the laser light. That is, an optical trap is taken to be a potential well with stiffness $k$. A spring with stiffness $\mathrm{k}$ prevents the mass attached to it from escaping and pulls it (or pushes it) back to the equilibrium position. Similarly, the optical trap tries to restore the particle to its equilibrium position, the bottom of the traps potential well [1]. There are many experimental works succesfully measured the stiffness of optical trap $[2,3,4]$. In those works, there are some methods used and their results confirm that the stiffness depends on laser intensity (or average laser input power). However, in medium the motion of particle is under impact not only of optical force, but of Brownian force, which depends on radius of particle and medium viscosity.

The goal of this article to simulate influence of principle parameters of optical trap on the stiffness.

\section{THEORY}

A pulsed Gaussian beam (PGB) is considered to trap fluctuating dielectric nano particles in the water plate (Fig.1). We consider the Gradient optical force is induced by 


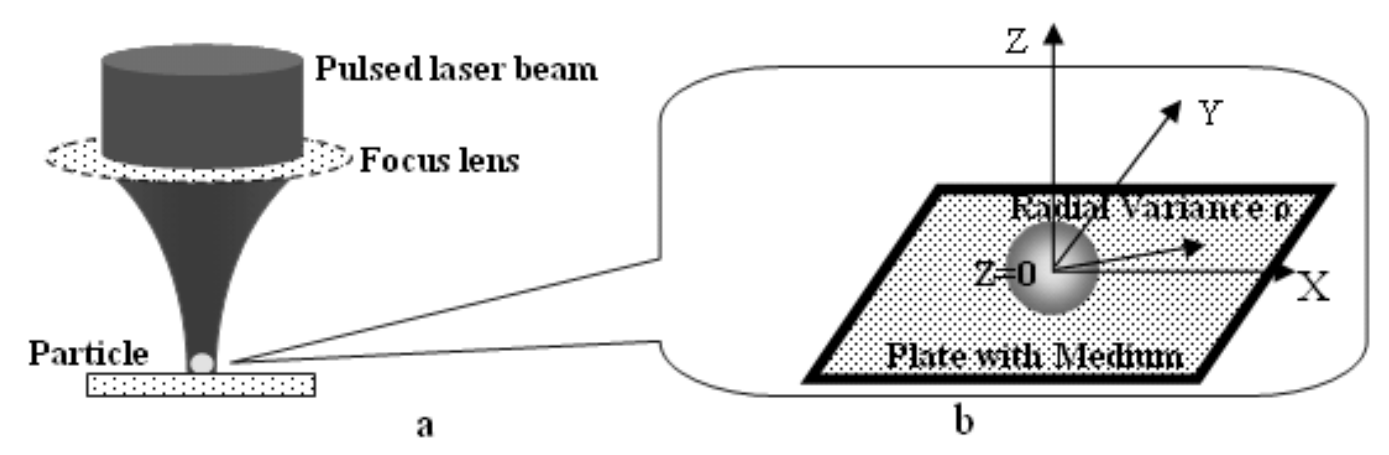

Fig. 1. (a) The schematic of optical tweezer. (b) The motion with radial variance of particle in the medium plate.

PGB acting on a Rayleigh dielectric particle. The polarization direction of the electric field is assumed to be along the $x$ axis.

The expression for the electric field of the above PGB is defined by [5]

$$
\begin{aligned}
\vec{E}(\rho, z, t)= & \hat{x} E_{0} \frac{i k w_{0}^{2}}{i k w_{0}^{2}+2 z} \exp \left\{-i\left[k(z)-\omega_{0} t\right]\right\} \\
& \times \exp \left[-i \frac{2 k z \rho^{2}}{\left(k w_{0}^{2}\right)^{2}+4 z^{2}}\right] \\
& \times \exp \left[-\frac{\left(k w_{0}^{2}\right)^{2} \rho^{2}}{\left(k w_{0}^{2}\right)^{2}+4 z^{2}}\right] \times \exp \left\{-\frac{[t-z / c]^{2}}{\tau^{2}}\right\}
\end{aligned}
$$

where $w_{0}$ is the spot radius of the beam waist at the plane $z=0, \rho$ is the radial coordinate, $\hat{x}$ is the unit vector of the polarization along the $x$ direction, $k=2 \pi / \lambda$ is the wave number, $\omega_{0}$ is the carrier frequency, $\tau$ is the duration of laser pulse. For the fixed input energy $U$ of a single beam, the constant $E_{0}$ is determined by $E_{0}^{2}=4 \sqrt{2} U /\left[w_{0}^{2}(\pi)^{3 / 2} \tau\right]$.

From the definition of the Poynting vector, we can readily obtain the intensity distribution

$$
I(\rho, z, t)=\langle\vec{S}(\rho, z, t)\rangle=\hat{z} \frac{P}{1+4 z^{2}} \exp \left[-\frac{2 \tilde{\rho}^{2}}{1+4 \tilde{z}^{2}}\right] \exp \left[-2\left(\tilde{t}-\frac{2 \tilde{z} k w_{0}^{2}}{c \tau}\right)\right]
$$

where $P=2 c \varepsilon_{0} \sqrt{2} U /\left[(\pi)^{3 / 2} w_{0}^{2} \tau\right]$. Here $\tilde{z}=z / k w_{0}^{2}, \tilde{t}=t / \tau$ ind $\tilde{\rho}=\rho / w_{0}$ are normalazea parameters.

For simplicity, we assume that the radius of the particle is much smaller than the laser wavelength $(a<<\lambda)$, ( ), that means the Rayleigh approximation is satisfied. In this case we can treat the dielectric particle as a point dipole. Assume that the refractive index of the particle is $n_{1}$ and $n_{1}>>n_{2}$, where $n_{2}$ is a refractive index of medium.

As shown in Fig.1 and definition of optical force [6], on the particle acts only the gradient force, $\vec{F}_{\text {grad }}$, which is given by

$$
\vec{F}_{\text {grad }, \rho}(\rho, z, t)=-\frac{\hat{\rho} 2 \beta I(\rho, z, t) \tilde{\rho}}{c n_{2} \varepsilon_{0} w_{0}\left(1+4 z^{2}\right)}
$$


where $\beta=4 \pi n_{2}^{2} \varepsilon_{0} a^{3}\left[\left(m^{2}-1\right) /\left(m^{2}+2\right)\right]$ is the polarizability, and $m=n_{1} / n_{2}[5,7]$.

Moreover, on the particle acts also Brownian force, which causes the particle moves randomly under the Brownian law. Assuming a low Reynold's number regime [8], the Brownian motion of the dielectric particle in the optical trap is described by a set of Langevin equations as follows:

$$
\dot{\overrightarrow{\gamma \rho}}(t)+K \vec{\rho}(t)=\sqrt{2 D} \gamma \vec{h}(t)
$$

where $\vec{\rho}(t)=[x(t), y(t)]$ is the dielectric particles position in the medium plate, $\gamma=6 \pi a \eta$ is its friction coefficient, $\eta$ is the medium viscosity, $\sqrt{2 D} \gamma \vec{h}(t)=\sqrt{2 D} \gamma\left[h_{x}(t), h_{y}(t)\right]$ is a vector describing the Brownian force, $D=k_{B} T / \gamma$ is the diffusion coefficient, $T$ is the absolute temperature, and $k_{B}$ is the Boltzmann constant, $K$ is the stiffness matrix.

On the other hand, in the presence of the optical force, equation (4) can be written in the form [9]:

$$
\dot{\overrightarrow{\gamma \rho}}(t)=F_{\text {grad }, \rho}(\vec{\rho}(t))+\sqrt{2 D} \gamma \vec{h}(t)
$$

From Eqs.(4) and (5) the total force acting on the particle

$$
F_{\text {grad, } \rho}(\vec{\rho}(t))=K \vec{\rho}(t)
$$

depends on the position of the particle itself.

We limit our analysis to a bidimensional system (i.e. considering $z=0$ ), because it is the most interesting from the applied point of view. Once the stiffness is known $\left(K_{\rho}\right)$, the position of the particle yields, for small displacements $(\Delta \rho(t))$, the force $\left(F_{\text {grad, } \rho}\right)$, i.e.

$$
F_{\text {grad, } \rho}(\rho, t)=K_{\rho} \Delta \rho(t)
$$

ffor bidimension system, where $\Delta \rho(t)$ is the mean value of radial variances experimentally measured in time interval $\Delta t$. For given particle (with radius a) and fluid (with constant drag coefficient $\eta$ ), stiffness $K_{\rho}$ increases linearly with increasing laser power of the optical trap. Using Eq. (7)) for our model, in which the laser field is the Gaussian pulse, the average stiffness $K_{\rho}$ over durating time of the laser pulse can be expressed by:

$$
\begin{aligned}
K_{\rho} & =\frac{\left\langle F_{\text {grad }, \rho}(\rho, t)\right\rangle_{t}}{\langle\Delta \rho(t)\rangle_{t}} \approx \frac{\left\langle F_{\text {grad }, \rho}(\rho, t)\right\rangle_{t}}{\langle\rho(t)\rangle_{t}} \\
& =\frac{\frac{1}{2 \tau} \int_{-\infty}^{\infty} F_{\text {grad }, \rho}(\rho(t), t) d t}{\frac{1}{2 \tau} \int_{-\infty}^{\infty} \rho(t) d t} \approx \frac{\frac{1}{2 \tau} \int_{-\tau}^{\tau} F_{\text {grad }, \rho}(\rho(t), t) d t}{\frac{1}{2 \tau} \int_{-\tau}^{\tau} \rho(t) d t}=\frac{\int_{-\tau}^{\tau} F_{\text {grad }, \rho}(\rho(t), t) d t}{\int_{-\tau}^{\tau} \rho(t) d t}
\end{aligned}
$$

As shown in previous work [10] the particle oscillates around the equilibrium position $(\rho=0)$ with an radial variance is about its radius. Thus in Eq. (8) the term $\langle\rho(t)\rangle_{t}$ can replace the term $\langle\Delta \rho(t)\rangle_{t}$.

Using Eqs. (3), (4) and (8), and numerical simulation, we can find the average stiffness, $K_{\rho}$, which depends on total energy $(U)$, beam waist $\left(w_{0}\right)$, and on duration $(\tau)$ of the Gaussian pulse, and on radius of particle $a$ with given parameters of surrounding medium $\eta, n_{2}, T$. 


\section{SIMULATED RESULTS AND DISCUSSION}

We compute the two-dimensional motion and the radial variance (position) of a glass particle in water (specimen at $z=0$ ) using the Brownian dynamic simulation method. A particle/bead- spring model is employed to represent the glass particle, and the following equation of motion is computed for each particle:

$$
\vec{\rho}(t+\delta t)-\vec{\rho}(t)=\frac{\vec{F}_{\text {grad }, \rho}(\vec{\rho}(t))}{\gamma} \times \delta t+\sqrt{2 D} \times \vec{h}(t) \times \delta t
$$

where $\delta t$ is the time increment of the simulation, $\vec{h}(t)$ is a random vector whose components are chosen from the range $[-1,1]$ in each time step. $\vec{F}_{\text {grad, },}(\vec{\rho}(t))$ in Eq. (9) describes the gradient optical force acting on the particle located at position $\rho(t)$. For example, at beginning time $t=0$, the glass particle is assumed to locate at the position $\rho(0)=w_{0}$, then we understand that the gradient optical force $F_{\text {grad, } \rho}\left(w_{0}, 0\right)$ acts on the particle, which will move to position $\rho(0+\delta t)=w_{0}+\Delta \rho$ after a time increment $\delta t$. . At this position the gradient force has new value $F_{\text {grad, } \rho}\left(w_{0}+\Delta \rho, 0+\delta t\right)$. Continously, after having $F_{\text {grad }, \rho}\left(\rho\left(t_{i}\right), t_{i}\right)$ and $\rho\left(t_{i}\right)$, using Newton- Contes integration method for two integrationss in (8) we have average stiffness $K_{\rho}$.

For numerical simulation we choose principle parameters as follows: $\lambda=1.064 \mu \mathrm{m}$, $m=n_{1} / n_{2}=1.592 / 1.332, \eta=7.797 \times 10^{-1}$ Pa.s, $k b T=4.14 \times 10^{-21}$ (the small glass particle and water, for instance) $[5], w_{0}=(0.5 \div 9.5) \mu m, a=(10 \div 50) \mathrm{nm}, \tau=(1 \div 20) \mathrm{ps}$, and the input total energy is changed by $U=(1 \div 200) \mu J$.

The influence of the total energy on average stiffness is simulated and illustrated in Fig.2. The results confirm again that stiffness of optical trap increases with increasing of the laser total energy. From Eqs.(2) and (3) can see that it is in good agreement to that presented in work of A. Kumar [2], in which the experimentally measured stiffness is directly proportional to the optical force.

Figures 3, 4, and 5 illustrate the influence of beam waist of laser beam, duration of laser pulse and radius of particle on stiffness, respectively on the stiffness. They show also that the stiffness depends not only on laser intensity, but mainly on the optical force, which depends on the beam waist, duration of the laser pulse. Moreover, the optical force depends on the polarizability, which depends on third power of particles radius, $a^{3}$. The dependence of the stiffness on principle parameters as shown in Fig.4 and Fig.5 is completely logical except what shown in Fig.3, where the stiffness reaches an optimal at $w_{0} \approx 1.06=\lambda$, increases and decreases with increasing of beam waist from $0.5 \mu \mathrm{m}$ to 1.06 $\mu \mathrm{m}$, and from $1.06 \mu \mathrm{m}$ to higher one, respectively.

This phenomena can be explained as follows: The beam divergence is directly proportional to the ratio between the wavelength $\lambda$, and the beam-waist diameter $2 w_{0}$ (i.e. $\left.\theta_{0}=2 \lambda / \pi 2 w_{0}\right)$. If the waist is squeezed, the beam diverges, which leads to obtain a low directional beam. Consequently, the depth of focus is directly proportional to the area of the beam at its waist, and inversely proportional to the wavelength (i.e. $2 b=2 \pi w_{0}^{2} / \lambda$ ). Thus when a beam is focused to a small spot size, the depth of focus is short and the plan of focus must be located with greater accuracy [10]. The low direction of beam and the short depth of focus causing by decreasing of beam waist lead to decreasing laser intensity 


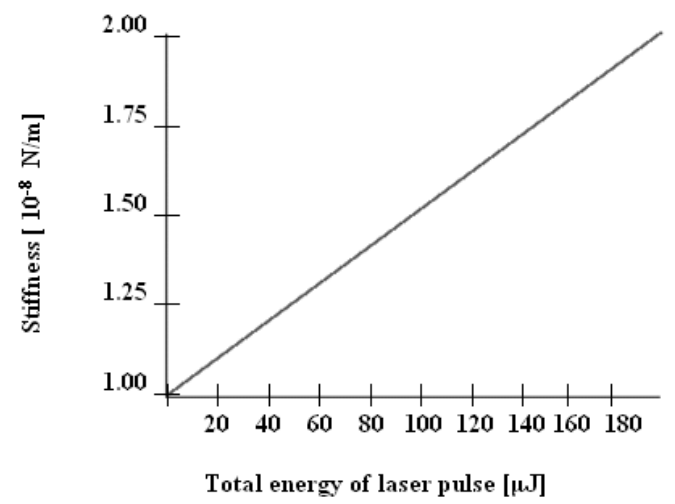

Fig. 2. Average stiffness vs total energy.

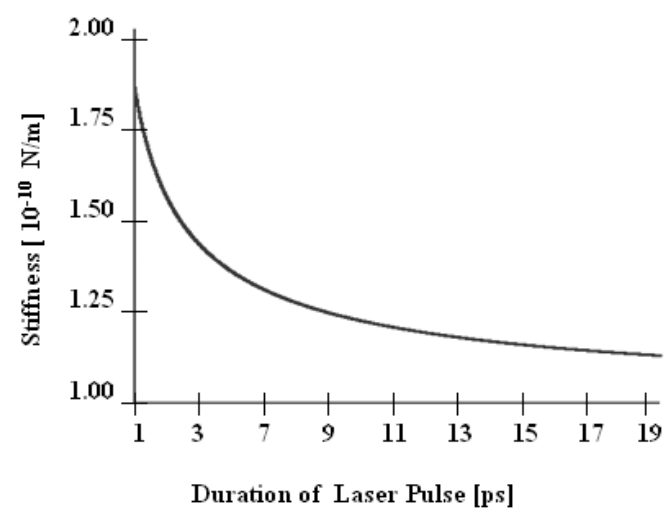

Fig. 4. Average stiffness vs. duration of pulse.

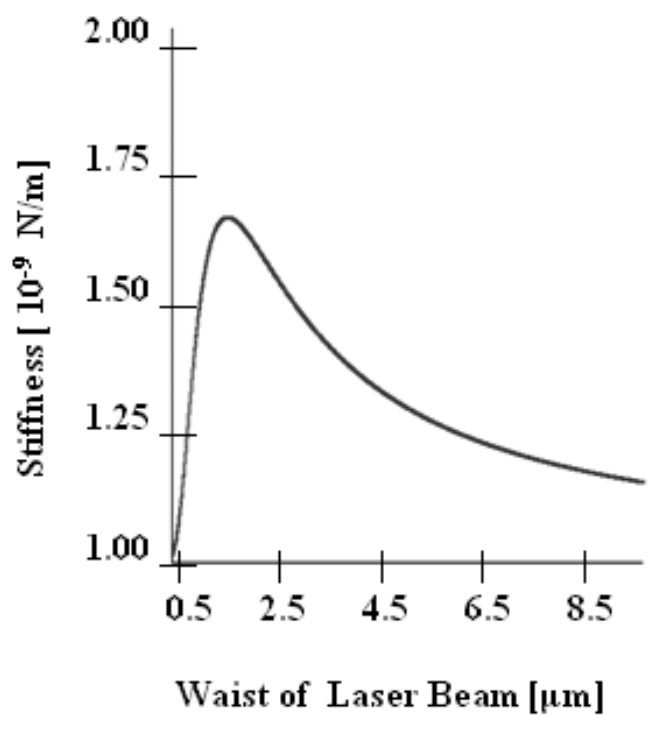

Fig. 3. Average stiffness vs beam waist.

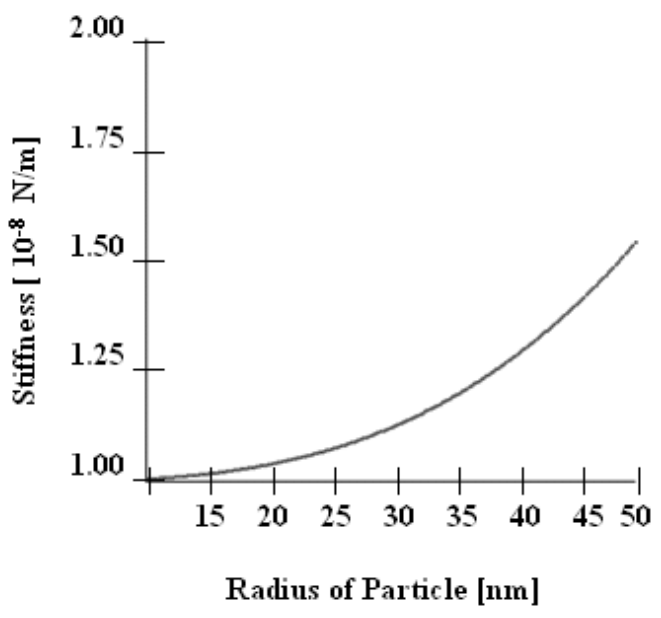

gradient in the area of the beam at its waist, consequently lead to decreasing of optical force.

\section{CONCLUSION}

From above discussions, we can collude that: First, the stiffness of opticae trap can be found by simulation using Langevin equation and expression of the gradient force; second, the average stiffness of trap does not directly depend on laser intensity only, but Sn the beam waist, total energy, duration of laser pulse, and on radius of trapped particle 
when using the laser Gaussian pulsed beam. The simulated results will be used to check the optical force or the radial variance of the particle, which are experimentally obtained.

\section{REFERENCES}

[1] ME/CE96, "Optical tweezer- Measuring the Steffness $k$ of the optical trap", http:/www.nbi.dk/ tweezer/introduction.htm (2005).

[2] A. Kumar De, D. Roy, B. Saha, and D. Goswami, A simple method for constructing and calibrating an optical tweezer, Current Science, 95 (2008) 723-724.

[3] D. Preece et al., Optics Express 17 (2009) 22718-22725.

[4] G. Honglian et al, Sciecce in China (Series A) 45 (2002) 919- 925.

[5] C. L. Zhao, L. G. Wang, X. H. Lo, Phys. Lett. A363 (2006) 502-506.

[6] A. Ashkin, J. M. Dziedzic, J.E. Bjorkholm, S. Chu, Opt. Lett. 11 (1986) 288-290.

[7] L.G. Wang et al., Opt. Lett. 32 (2007) 1393-1395.

[8] J. Happel and H. Brenner, Low Reybnold Number Hydrodynamics, Springer, NEW York, 1983, Chap. 4.

[9] G. Volpe, G. Volpe, and D. Petrol, Phys. Rev. E76 (2007) 061118.

[10] H. Q. Quy et al., Chinese Optics Letters 8 (3) (2010) 332-334.

Received 13 July 2010. 